\title{
Direct experimental observation of two-dimensional shrinkage of the exciton wave function in quantum wells
}

\author{
Yasuaki Masumoto \\ The Institute for Solid State Physics, The University of Tokyo, Roppongi, Minato-ku, Tokyo 106, Japan \\ Mitsuru Matsuura \\ Department of Applied Science, Faculty of Engineering, Yamaguchi University, Tokiwadai, Ube 755, Japan \\ Seigo Tarucha and Hiroshi Okamoto \\ Musashino Electrical Communication Laboratory, Nippon Telegraph and Telephone Corporation, \\ Midori-machi, Musashino-shi, Tokyo 180, Japan
}

(Received 1 July 1985)

\begin{abstract}
We have studied the oscillator strength of the lowest $1 s$ heavy excitons in GaAs-AlAs quantum wells as a function of well-layer thickness by means of optical absorption. The oscillator strength of the lowest $1 \mathrm{~s}$ heavy excitons is largely enhanced with the decrease of well-layer thickness. The result is the first full experimental observation of two-dimensional shrinkage of the exciton wave function in quantum wells.
\end{abstract}

It is interesting to clarify experimentally the form of the exciton wave function in semiconductor quantum wells, both from the viewpoint of general physics as well as from that of semiconductor physics. This is because excitons in quantum wells are excellent analogues of hydrogen atoms in one-dimensional square wells. ${ }^{1}$ By reducing the well thickness, we can continuously change the wave functions of excitons or hydrogen atoms from the three-dimensional to the two-dimensional state. Recent advances in semiconductor technology allow us to study experimentally this dimensional problem for excitons by creating quantum wells whose thickness is comparable to the exciton Bohr radius. The effect of dimensionality on the oscillator strength of the exciton $^{2-4}$ as well as on the binding energy of the exciton has been extensively studied by theorists. ${ }^{2-6}$ The recent magnetoabsorption experiment has revealed the binding energy of excitons in quantum wells. ${ }^{7,8}$ However, there has been no full experimental study of the oscillator strength.

Compared with the binding energy, the oscillator strength is a more direct probe for the investigation of the nature of the wave function of excitons. This is because the oscillator strength is directly proportional to the square of the amplitude of the exciton wave function at the point where the two constituents of the exciton, an electron and a hole, overlap. It is expected that the overlap amplitude of the electron and the hole rapidly increases with the decrease of the well-layer thickness $L_{z}$, when $L_{z}$ is smaller than the exciton Bohr radius $a_{B}$. Then the oscillator strength of excitons will rapidly increase. Some authors have reported the enhancement of the exciton oscillator strength with the decrease of well-layer thickness by comparing the lifetime of excitons. 9,10 However, the lifetime of excitons does not always reflect the oscillator strength, owing to the considerable contribution of the nonradiative processes. In fact, the observed reduction of the lifetime is too small compared with the enhancement of the oscillator strength directly presented in this paper. ${ }^{11}$ In this work, we systematically study the oscillator strength of the lowest $1 s$ heavy excitons in GaAs-AlAs quantum wells as a function of well thickness by means of optical absorption. The results give the first full experimental observation of the nature of the exciton

wave function in quantum wells.

In this work ten multi-quantum-well samples grown by molecular-beam epitaxy on GaAs(100) substrates were used. Their characters are listed in Table I. Samples (GaAs-AlAs, QW1-QW10) consist of alternate undoped GaAs well layers and undoped AlAs barrier layers. The thickness of the GaAs well layers ranges from 30 to $254 \AA$. A hole in the GaAs substrate of all the samples was made by means of selective chemical etching. Samples were directly immersed in superfluid helium. For the opticalabsorption measurement, an incandescent lamp and a $50-\mathrm{cm}$ monochromator were used. The light beam was carefully set to pass vertically through the hole in each GaAs substrate and the quantum-well layers.

In Fig. 1 absorption spectra $\alpha(E)$ of six representative samples are shown. Here the absorption coefficient is defined as $\alpha(E)=-\ln \left(I / I_{0}\right) / d(\mathrm{GaAs})$, where $I_{0}$ and $I$ are the incident and transmitted light intensities, respectively. The total thickness of the GaAs, $d(\mathrm{GaAs})$, is equal to the well-layer thickness $L_{z}$ multiplied by the number of GaAs

TABLE I. List of samples. $L_{z}$ is the GaAs well thickness, $L_{b}$ the AlAs barrier thickness, $d(\mathrm{GaAs})$ the total thickness of $\mathrm{GaAs}$, and $E_{n=1}$ the energy of the absorption peak due to the lowest $1 s$ heavy-exciton state at $2.0 \mathrm{~K}$.

\begin{tabular}{lcccc}
\hline $\begin{array}{c}\text { GaAs-AlAs } \\
\text { sample numbers }\end{array}$ & $\begin{array}{c}L_{z} \\
(\AA)\end{array}$ & $\begin{array}{c}L_{b} \\
(\AA)\end{array}$ & $\begin{array}{c}d(\mathrm{CaAs}) \\
(\mu \mathrm{m})\end{array}$ & $\begin{array}{c}E_{n=1} \\
(\mathrm{eV})\end{array}$ \\
\hline QW1 & 30 & 40 & 0.30 & 1.845 \\
QW2 & 43 & 62 & 0.86 & 1.683 \\
QW3 & 53 & 50 & 1.18 & 1.672 \\
QW4 & 58 & 71 & 0.99 & 1.642 \\
QW5 & 76 & 33 & 2.08 & 1.622 \\
QW6 & 83 & 93 & 1.43 & 1.579 \\
QW7 & 96 & 91 & 0.96 & 1.571 \\
QW8 & 108 & 36 & 2.63 & 1.572 \\
QW9 & 154 & 131 & 2.00 & 1.540 \\
QW10 & 254 & 122 & 2.03 & 1.524 \\
\hline \hline
\end{tabular}



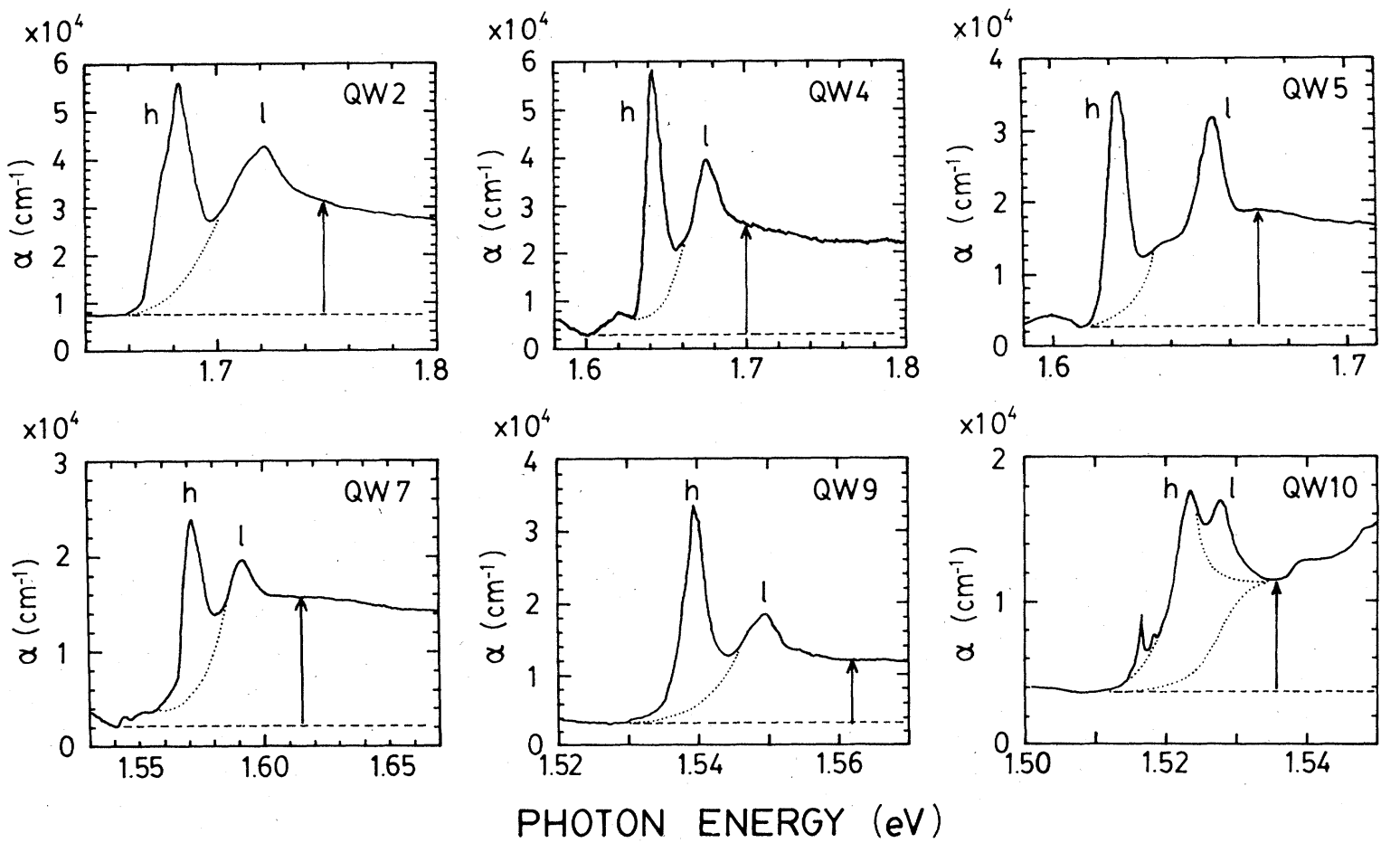

FIG. 1. Absorption spectra of the lowest heavy and light excitons in six representative GaAs-AlAs multi-quantum-well samples at $2.0 \mathrm{~K}$. Heavy and light excitons are denoted by $h$ and $h$, respectively. The background comes from the reflection loss, which is shown by dashed lines. Absorption areas of the heavy excitons plotted in Fig. 2 are those surrounded by solid lines and dotted lines. Absorption coefficient at the continuum plotted in the inset of Fig. 2 are indicated by arrows.

well layers. Observed absorption peaks located at the lowest energy are ascribed to the $1 s$ heavy exciton $(n=1, e-\mathrm{HH})$, which is composed of an electron and a heavy hole belonging to the lowest state $(n=1)$ in the quantum well. The absorption peaks located at the higher energy are ascribed to the $1 s$ light exciton $(n=1, e-\mathrm{LH})$, composed of an electron and a light hole. The absorption spectra are steplike above the $1 s$ light-exciton energy. They correspond to the continuum transition from both the heavy and light valence bands to the conduction band. Steplike absorption spectra reflect the steplike two-dimensional density of states of the conduction and valence bands in a quantum well. The absorption coefficient at the continuum-band positions increases with the decrease of well-layer thickness $L_{z} \cdot{ }^{12}$. Simultaneously, the integrated areas of the absorption peaks due to heavy and light excitons increase.

The integrated areas of the absorption peaks are directly proportional to the oscillator strength $f$ as follows:

$$
\int_{E_{1}}^{E_{2}} \alpha(E) d E=\frac{2 \pi^{2} e^{2} \hbar}{m_{0} c \sqrt{\epsilon_{b}}} f
$$

where $m_{0}$ is the mass of electron, $e$ is the charge of electron, $c$ is the light velocity, $\epsilon_{b}$ is the background dielectric constant, and the lower and upper limits of the integral, $E_{1}$ and $E_{2}$, cover the absorption peaks. Thus, we can directly estimate the oscillator strength $f$ from the absorption area. The oscillator strength $f$ of excitons in quantum wells is considered to vary spatially, because quantum wells consist of many islandlike clusters whose thickness fluctuates by the height of a monolayer. ${ }^{13}$ The exciton in each cluster has the oscillator strength for that cluster. Therefore, the oscillator strength $f$ in Eq. (1) is regarded as the spatially averaged one.

In Fig. 2, the obtained absorption areas of the heavyexciton peaks are shown as a function of $L_{z}$. In the inset of Fig. 2, the absorption coefficients at the continuum positions indicated by arrows in Fig. 1 are plotted. ${ }^{14}$ From Fig. 2 , one can find that the absorption coefficient at the continuum and the absorption area of the heavy-exciton peaks are strongly enhanced with the decrease of $L_{z}$. The absorption coefficient is proportional to $1 / L_{z}$, while the absorption area of heavy-exciton peaks is enhanced much more with the decrease of $L_{z}$.

From the same variational calculation described previous$1 y$, we can obtain the oscillator strength of $1 s$ excitons in an infinite square well. ${ }^{3,4}$ Dropping the plane-wave term, we take the following trial function for the $1 \mathrm{~s}$ exciton wave function:

$$
\begin{aligned}
\Psi= & N \cos \left(\pi z_{e} / L_{z}\right) \cos \left(\pi z_{h} / L_{z}\right) \\
& \times \exp \left\{-\left[\alpha^{2}\left(x^{2}+y^{2}\right)+\beta^{2}\left(z_{e}-z_{h}\right)^{2}\right]^{1 / 2}\right\} .
\end{aligned}
$$

Here two variational parameters $\alpha$ and $\beta$ are related to the spatial extent of the exciton in directions parallel $(x, y)$ and perpendicular $(z)$ to the well layer, respectively. The quantum well is assumed to have infinite wall at $z= \pm L_{z} / 2$ and the $z$ coordinates of the electrons and holes are $z_{e}$ and $z_{h}$, respectively. $N$ is determined by the normalization condition. This trial function contains that used by Bastard, Mendez, Chang, and Esaki as a special case. ${ }^{5}$ The oscillator 


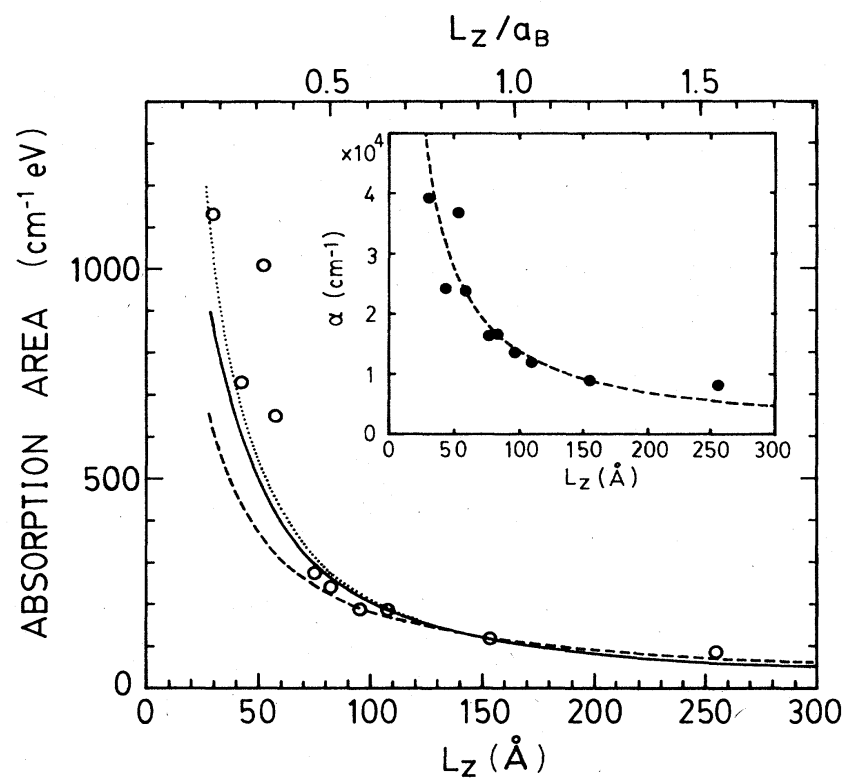

FIG. 2. $L_{z}$ dependence of the absorption area (O) of the lowest $1 s$ heavy exciton. The solid and dotted lines are the oscillator strength calculated on the basis of Eq. (3) with and without taking account of the energy dependence of the optical transition, respectively. The dashed line shows the $1 / L_{z}$ dependence. Calculated lines are normalized to fit the experimental data of QW9. In the inset the $L_{z}$ dependence of the absorption coefficient $(\bullet)$ at the continuum indicated by arrows in Fig. 1 is plotted. The dashed line shows $1 / L_{z}$ dependence.

strength of $1 s$ excitons is calculated by

$$
f=\left(L_{z} N^{2} / 2 m_{0} \hbar \omega\right)\left|M_{c v}\right|^{2} F\left(k_{z} L_{z} / 2\right),
$$

where $M_{c v}$ is the matrix element of the optical transition from the valence to the conduction band, $\hbar \omega$ is the optical transition energy, and $k_{z}$ is the $z$ component of the photon wave vector in the well layers. A factor $F(x)$ $=\left[(\sin x / x) \pi^{2} /\left(x^{2}-\pi^{2}\right)\right]^{2}$ arises from the wave-vector mismatch between excitons and photons. Because the relation $k_{z} L_{z} / 2<0.35$ holds in all the samples, it is exact enough to regard $F\left(k_{z} L_{z} / 2\right)$ in Eq. (3) as 1.0 for the analysis of the experiment. As $L_{z}$ approaches zero, Eq. (3) converges to $\left(4 \alpha^{2} / \pi m_{0} \hbar \omega L_{z}\right)\left|M_{c v}\right|^{2}$. In the limit of small $L_{z}, \alpha$ goes to $2 / a_{B}$. Then expression (3) agrees with the well-known formula $\left(16 / \pi m_{0} \hbar \omega L_{z} a_{B}^{2}\right)\left|M_{c v}\right|^{2}$ for the twodimensional exciton. ${ }^{2}$ As is shown by Chang and Schulman, ${ }^{15}$ the square of the optical matrix element $\left|M_{c v}\right|^{2}$ can be regarded as independent of $L_{z}$ in the present range (30 A $\left.<L_{z}<254 \AA\right)$. Thus we calculated the relative oscillator strength regarding the term $\left|M_{c v}\right|^{2}$ in Eq. (3) as a constant. The calculated oscillator strength is shown as a function of $L_{z}$ by solid and dotted lines in Fig. 2 . The solid and dotted lines are calculated with and without taking into account the energy dependence of the optical transition energy $\hbar \omega$ ( $=E_{n=1}$ in Table I), respectively. Here we have adopted the values of static dielectric constant $\epsilon_{0}=12.5$ and the reduced mass of exciton in the $x, y$ directions $\mu=0.04 m_{0}$, so that the Bohr radius $a_{B}=\epsilon_{0} \hbar^{2} / \mu e^{2}$ is taken to be $165 \AA$. The calculated curves are normalized to fit the absorption area of the $1 s$ heavy exciton in the QW9 sample. As a result of normalization, the calculated curves depend little on the values of $\epsilon_{0}, \mu$, and $a_{B}$. The agreement between the experimental values and the calculated curve is fairly good unless the well thickness is thinner than $60 \AA$. It is noted that almost the same calculated curves have been obtained even with other forms of trial functions by Bastard et al. ${ }^{5}$ or Greene and Bajaj, ${ }^{6}$ although the present trial function in Eq. (2) gives the largest binding energy for excitons.

It is instructive to compare the $L_{z}$ dependence of the absorption area of the heavy-exciton peaks with that of the absorption coefficient at the continuum. With the decrease of $L_{z}$, the absorption coefficient grows larger in proportion to $1 / L_{z}$. This dependence comes from the fact that the square of the overlap amplitude of the wave functions of the electrons and holes is proportional to $1 / L_{z}$, because the unbound states of electrons and holes are confined in the well of thickness $L_{z}$ in the $z$ direction but are extended in the $x, y$ directions, while the $1 s$ exciton wave function shrinks in both the $z$ and $x, y$ directions. In the $z$ direction, the exciton wave function shrinks in proportion to $L_{z}$, while in the $x, y$ directions the exciton wave function shrinks in proportion to $1 / \alpha$. In the limit of small $L_{z}, 1 / \alpha a_{B}$ goes to $\frac{1}{2}$. This fact explains the more drastic increase of the absorption area of the heavy-exciton peak compared with the increase of the absorption coefficient at the continuum. Thus, the enhancement character of absorption area presents direct experimental verification of the two-dimensional shrinkage of the exciton wave function in quantum wells. In the case that the well layer is thinner than $60 \AA$, the experimental values are much larger than the calculated ones. The present experiment presents the relative increase of the oscillator strength of excitons in quantum wells. The calculated curve varies little even if we change the value of the Bohr radius $a_{B}$ from 100 to $200 \AA$. This is because the calculated curve is normalized to fit the experimental data of the QW9 sample. Thus the disagreement between experiment and calculation is unavoidable.

The experimental data definitely show that the exciton wave function shrinks much more than calculated for the infinite-well model. This deviation is enhanced even more if we take the finite-well model. Then the exciton wave function does not shrink as much with the decrease of $L_{z}$, because the wave function penetrates somewhat into the barrier layer. The effect of the image force on excitons somewhat enhances the oscillator strength with the decrease of $L_{z}$. However, the enhancement explains only a part of the deviation. ${ }^{16}$ If we assume that the reduced mass $\mu$ of the exciton is enhanced by $\approx 20 \%$ below $L_{z} \approx 60 \mathrm{~A}$, the oscillator strength increases by $\approx 40 \%$, so that the deviation is canceled. This is because the Bohr radius $a_{B}$ is proportional to $1 / \mu$, and because the oscillator strength of the twodimensional exciton is enhanced in proportion to the enhancement of $1 / a_{B}^{2}$. In this connection, we note the recent magneto-optical experiments. ${ }^{7,8}$ The enhancement of the reduced mass of excitons deduced from our present experiment qualitatively agrees with that deduced from the magneto-optical experiments. The nonparabolicity of the heavy-hole subband probably explains the increase of the reduced mass with the decrease of $L_{z}$, because the hole states with larger wave vectors contribute to the exciton wave function with its shrinkage.

In summary, the oscillator strength of the lowest $1 s$ heavy excitons in GaAs-AlAs quantum wells is studied as a function of well-layer thickness by means of optical absorption. 
The oscillator strength of the lowest $1 s$ heavy excitons is rargely enhanced with the decrease of well-layer thickness. The enhancement character gives the full experimental verification of two-dimensional shrinkage of the exciton wave function in quantum wells. The exciton wave function shrinks much more than the calculated one when the quantum well is thinner than $60 \AA$. This fact suggests that the reduced mass of excitons is then enhanced by $\approx 20 \%$.
The authors wish to thank Professor M. Matsuoka for fruitful discussions and continuous encouragement. This work was in part supported by the Scientific Research Grant-in-Aid No. 59460024 and the Scientific Research Grants-in-Aid No. 60222017 and No. 60222033 for Special Project Research on "Alloy Semiconductor Electronics" from the Ministry of Education, Science and Culture of Japan.
${ }^{1}$ R. Dingle, in Festkörperprobleme, edited by H. J. Queisser, Advances in Solid State Physics, Vol. XV (Pergamon, New York, 1975), p. 21.

${ }^{2}$ M. Shinada and S. Sugano, J. Phys. Soc. Jpn. 21, 1936 (1966).

${ }^{3}$ Y. Shinozuka and M. Matsuura, Phys. Rev. B 28, 4878 (1983); 29, 3717(E) (1984).

${ }^{4}$ M. Matsuura and Y. Shinozuka, J. Phys. Soc. Jpn. 53, 3138 (1984).

${ }^{5}$ G. Bastard, E. E. Mendez, L. L. Chang, and L. Esaki, Phys. Rev. B 26, 1974 (1982).

${ }^{6}$ R. L. Greene and K. K. Bajaj, Solid State Commun. 45, 831 (1983).

${ }^{7}$ S. Tarucha, H. Okamoto, Y. Iwasa, and N. Miura, Solid State Commun. 52, 815 (1984).

8 J. C. Maan, G. Belle, A. Fasolino, M. Altarelli, and K. Ploog, Phys. Phys. Rev. B 30, 2253 (1984).

${ }^{9}$ E. O. Göbel, H. Jung, J. Kuhl, and K. Ploog, Phys. Rev. Lett. 51, 1588 (1983).

10J. Christen, D. Bimberg, A. Steckenborn, and G. Weimann, Appl. Phys. Lett. 44, 84 (1983).

${ }^{11}$ The ratio of the exciton lifetime in two samples, 2.85 , observed by Göbel et al. (Ref. 9) is too small compared with the theoretical value, 3.8 , and the experimentally observed ratio, 5.5 , of the oscillator strength of excitons which are shown in Fig. 2. A similar conclusion holds for the result of Christen et al. (Ref. 10).

${ }^{12} \mathrm{H}$. Iwamura, H. Kobayashi, and H. Okamoto, Jpn. J. Appl. Phys. 23, L795 (1984).
${ }^{13}$ C. Weisbuch, R. Dingle, A. C. Gossard, and W. Wiegmann, Solid State Commun. 38, 709 (1981).

${ }^{14}$ The absorption area of heavy excitons and the continuum-band positions shown by arrows in Fig. 1 are determined by the following guidelines. (1) The absorption line profile of heavy excitons is determined so as to be symmetric. (2) The absorption line profile of heavy excitons and that of light excitons are assumed to be the same because a common origin, that is, fluctuation of the well-layer thickness broadens these absorption lines. The continuum absorption coefficient is estimated at the positions where the contribution of absorption due to $1 s$ light excitons is negligible. The continuum absorption coefficient thus estimated corresponds to the sum of absorption coefficients of heavy and light continuum bands. If we adopt other guidelines, the estimated absorption area of heavy excitons differs from the present value at most by $\approx 25 \%$. Then, however, the relative values normalized by the value of the QW9 sample vary at most by $\approx 10 \%$. Thus, the conclusion of this paper does not depend on the selection of guidelines.

${ }^{15}$ Y. C. Chang and J. N. Schulman, Appl. Phys. Lett. 43, 536 (1983); Phys. Rev. B 31, 2069 (1985).

${ }^{16} \mathrm{M}$. Matsuura (unpublished); the calculated oscillator strength of excitons for $L_{z}=50 \AA$ normalized by that for $L_{z}=154 \AA$ increases by about $10 \%$, taking into account the effect of image force. 